\title{
Towards Bio-impedance Based Labs: A Review
}

Pablo Pérez ${ }^{1,2}$, Andrés Maldonado ${ }^{1,3}$, Alberto Yúfera ${ }^{1,2}$, Gloria Huertas ${ }^{1,3}$, Adoración Rueda ${ }^{1,3}$ and José Luis Huertas ${ }^{1,3}$

1. Instituto de Microelectrónica de Sevilla, Universidad de Sevilla, CSIC, Sevilla 41092, Spain

2. Dpt. Electronic Technology, ETSII, Universidad de Sevilla, Sevilla 41012, Spain

3. Dpt. Electronic and Electromagnetism, F. Physics, Universidad de Sevilla, Sevilla 41012, Spain

\begin{abstract}
In this article, some of the main contributions to BI (Bio-Impedance) parameter-based systems for medical, biological and industrial fields, oriented to develop micro laboratory systems are summarized. These small systems are enabled by the development of new measurement techniques and systems (labs), based on the impedance as biomarker. The electrical properties of the life mater allow the straightforward, low cost and usually non-invasive measurement methods to define its status or value, with the possibility to know its time evolution. This work proposes a review of bio-impedance based methods being employed to develop new LoC (Lab-on-a-Chips) systems, and some open problems identified as main research challenges, such as, the accuracy limits of measurements techniques, the role of the microelectrode-biological impedance modeling in measurements and system portability specifications demanded for many applications.
\end{abstract}

Key words: Bio-impedance sensing, signal conditioning circuits, microelectrode, OBT (Oscillation Based Test), LoC.

\section{Introduction}

In 1993, Andreas Manz [1] developed a device capable of performing capillary electrophoresis on a single chip, first of its kind. The chip was generated using the know-how from microelectronic fabrication. Devices like this were named Micro Total Analysis Systems (uTAS), the term was coined by Andreas Manz itself. Soon, after the initial development, scientist realized that the original concept was applicable on a larger scale than previously though. The functionality provided by the downscaling of many macro world machines; reactors, separators, fluid-handling devices, etc. could possibly lead to a "LoC (Lab-on-a-Chip)" based experimental science in the related fields of knowledge, to know; medicine, biology, chemistry, etc. [2].

LoC systems are currently developed as an alternative to acquire biological parameters, mimicking the protocols or alternatively enabling new assays techniques to substitute the weighted and huge

Corresponding author: Pablo Pérez, M.Sc. in computer engineering, research fields: biomedical circuits and systems. classical biolab systems, avoiding the expensive material and the required human cost, and reducing the excessive time consumption [3]. The LoC paradigm aims at benefiting from mature technology existing in several fields: biology, medical, chemical, engineering (electrical, mechanical, etc.), microelectronics, computer science, etc., to solve a measurement problem in the bio-medical one. The system input is defined from a bio-signal transduction occurring in a sensor. Sensors can be classified according to their unique nature: optical, mechanical, acoustic, chemical or electrical. Many optical assays are being migrated to LoC systems since optical sensors technology has proven itself as reliable in the microelectronic technology. This can serve the large number of optical assays being carried out on biomedical labs [4-7].

There exists evidence for further research and development on LoC technology. The biomedical engineering field comprises all the sensors and applications depicted in Table 1. Most of the applications are yet in an early state of maturity to support health attention in major health systems. Despite 
Table 1 Bio devices nature and applications.

\begin{tabular}{|c|c|c|}
\hline Sensor nature & Applications & Ref. \\
\hline Optical & Handheld fluorometers & {$[10]$} \\
\hline Mechanical & $\begin{array}{l}\text { Triglyceride biosensor } \\
\text { C-Reactive protein detection } \\
\text { Glucose level analysis }\end{array}$ & [11] \\
\hline Acoustic & Hearing aid devices & \\
\hline Chemical & $\begin{array}{l}\text { Chemical properties } \\
\text { or compound detectors }\end{array}$ & $\begin{array}{l}{[12]} \\
{[8]}\end{array}$ \\
\hline Electrical & $\begin{array}{l}\text { Blood cell counting } \\
\text { Stress detection } \\
\text { Glucose concentration } \\
\text { Tissue impedance evolution } \\
\text { Cell-growth tax } \\
\text { Toxicological analysis } \\
\text { Bacterial detection }\end{array}$ & $\begin{array}{l}{[13]} \\
{[14]} \\
{[15]}\end{array}$ \\
\hline Device nature & Applications & Ref. \\
\hline Microfluidics & $\begin{array}{l}\text { DNA sequencing and analysis } \\
\text { Drug delivery and guidance }\end{array}$ & [16] \\
\hline Complex analysis & $\begin{array}{l}\text { Big data pool analysis in the health domain } \\
\text { Sensor fusion }\end{array}$ & $\begin{array}{l}{[11]} \\
{[17]}\end{array}$ \\
\hline Stimulation & $\begin{array}{l}\text { Spinal cord stimulation } \\
\text { Drug delivery and guidance }\end{array}$ & {$[18]$} \\
\hline
\end{tabular}

this fact, the great potential of this technology lies around the possibility to provide PoC (Point-of-Care) diagnosis. A good example for this would be blood cell counting technology $[8,9]$. This could be used to diagnose certain conditions, which may be expressed via the blood properties in our body.

The possibilities for this field of technology are endless. Electroderm analysis [9] can be used to diagnose stress related conditions. Moreover, many optical assays are migrating to LoC systems since optical sensors technology is well established by the microelectronic technology. Large number of optical assay has been done traditionally at biomedical labs [10]. Google itself has patented a smart contact lens to continuously measure glucose levels in tears. Tear glucose level is directly related to blood glucose level [20]. In addition, for sensing applications, biomedical engineers can take advantage of existing technologies in microelectronic devices. Further application of MEMs devices includes micro-actuators which, for instance, can store and deliver drug when necessary [16].

In 1957, Schwan, analyzed for the first time, the dielectric properties of biological matter [21]. He realized both dielectric constant $(\varepsilon)$ and conductivity $(\sigma)$ were varying as a function of the signal frequency. Biological electrical impedance or simply BI (Bio-Impedance) represents the electrical response of biological mater to electric fields. This response, if measured, can provide some insight on several properties (and some more under research). The present work is focused on electrical transducers, specifically, those occupied to detect the electrical properties of the biological mater, aiming at monitoring a biomarker representative of a biological process. The impedance $\left(Z_{x}\right)$, is composed by a resistive $\left(R_{x},\right)$ and a reactive $\left(C_{x}\right)$ components.

Currently, many research goals are focused on measuring the impedance of biological samples. Several are the major benefits of measuring impedances in medical and biological environment. Firstly, most biological parameters and processes can be monitored using its impedance as marker. Secondly, it is important to remark that, in many contexts, the bio-impedance measurement is a non-invasive technique. Finally, it represents an alternative cheap technique to labs; comparing with classical biological laboratories assay, those based on signaling and 
labeling. It is very important to remark that traditional laboratories do offer high cost techniques for detecting bacterial elements in samples. The bio-impedances detection method can help to trace these elements [15]. Main applications identified in the literature are summarized in Table 2.

In order to address the measurement problem for any bioimpedance $Z_{x}$, with magnitude $Z_{x o}$ and phase $\phi$, an illustration is provided in Fig. 1. It is necessary to provide an ac signal, where $\omega$ represents the signal frequency. This signal is used to excite the sample under test, $Z_{x}$, and then, the response, current or voltage, is picked-up by a trans-impedance amplifier or voltage amplifier. Processing circuits must extract the useful information $\left(Z_{x 0}, \phi\right)$ from measurement, being in most cases necessary to establish a synchronization with the AC excitation circuits to guarantee the accuracy of the technique. Signals are coming in and out from the bio-samples travel along the electrodes. In this case, electrodes operate as the transducers converting electronic charge, at circuits, into ionic charge (at biological environment) and vice versa. Due to electrical properties of electrodes, both, the excitation and processing circuits, will be influenced by electrode frequency response, hence knowledge of the electrode-electrical model is a requirement for an adequate circuit design.

In general, for impedance measurement process based on electrodes, the first specification to be considered is the electrical model employed for both the electrode and biology systems [46]. This fact must be considered along with the second one: the definition of the algorithm implemented by the measurement technique. This is one of the main drawbacks on the excitation circuit design, which is demanded by the need of using electrodes and its electrical response, frequency dependent, with a wide range of impedances values depending on the working frequency (ranging from several ohms to values in the $\mathrm{M}$ range, for very small microelectrodes). Also, applied voltage to electrodes must be amplitude limited to guarantee its correct linear biasing region, generally some tens of $\mathrm{mV}$. It must be said that, for impedance measurement, the electrodes are usually on the path of the ac signal applied, so electrode models will influence eventually on the measurement.

Table 2 Applications in the biomedical domain.

\begin{tabular}{|c|c|c|}
\hline Sample & Applications & References \\
\hline \multirow{2}{*}{ Tissue } & Myocardial ischemia & {$[13,22]$} \\
\hline & Tissue degradation & {$[23,24]$} \\
\hline \multirow{4}{*}{ Cell Level } & Cellular growth & {$[14,25-29]$} \\
\hline & Impedance spectroscopy & {$[30,31]$} \\
\hline & Bacterial detection & {$[15,26]$} \\
\hline & Cancer & {$[32-35]$} \\
\hline \multirow{3}{*}{$\begin{array}{l}\text { Bio-marker } \\
\text { or assay }\end{array}$} & Toxicological analysis & {$[15]$} \\
\hline & Glucose concentration & [36] \\
\hline & DNA detection & {$[37,38]$} \\
\hline \multirow{3}{*}{ Body } & EIT (Electr. Imp. Tomography) & {$[39,40]$} \\
\hline & ECG (Electrocardiography) EEG (Electroencephalography) SCS (Spinal Cord Stimulation) & {$[18,41-43]$} \\
\hline & Respiration monitoring & {$[44,45]$} \\
\hline
\end{tabular}

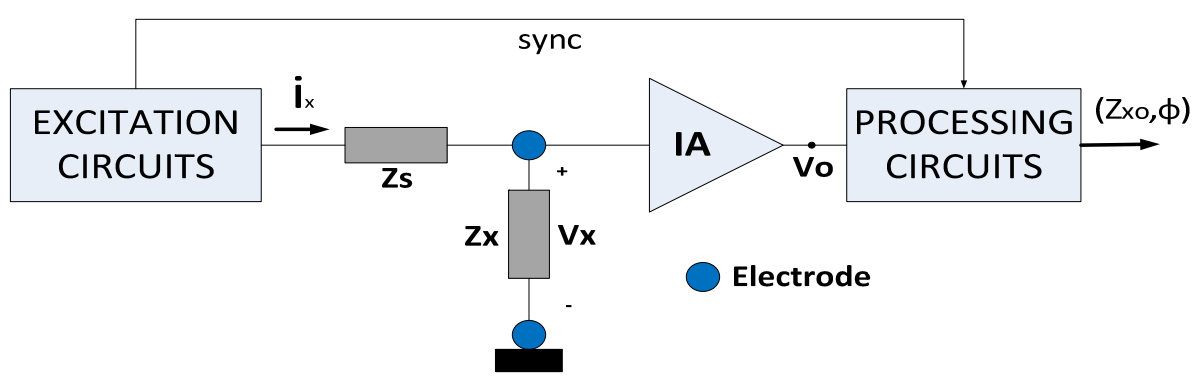

Fig. 1 General block diagram for the measurement of $Z_{x}$ impedance. 
A second problem to be solved is the selection of the measurement technique to be implemented. In the literature, many methods have been reported [47]. Commonly, most of them require excitation and processing circuits. Excitation is usually done with AC current sources, while processing steps relies on algorithmic approaches, in order to decode the sample voltage response to signal excitation, the mission of the system described would be the effective determination of both bio-impedance components. Classical approaches consist on coherent demodulation principle [48] or synchronous sampling [49], leading, in general, to good results. In both techniques, processing circuits must be synchronized with input signals. This is a major requirement for the techniques to work. Best noise performance is achieved when proper filter functions (HP and LP) are implemented on the design. A general block diagram is illustrated in Fig. 1. For both algorithms, the signal synchronization is mandatory. These measurement principles work as feed-forward systems: the signal generated on $Z_{x}$ is amplified, and then processed using appropriate electronics for that purpose.

Many of the actual applications for biomedical measurements have the potential to support real time monitoring, supervision and therapeutic role. The design of these systems has also others requirements; low-weight, small-size, low-power consumption and reliable and secure data transfer. Micro-electromechanical, micro-fluidic, micro-electronic and others micro-technologies are being adopted to this meet these requirements. It is important to remark that current microelectronic technology scale is similar to biological dimensions where the bio-chemical life processes are occurring and hence new biomedical methodologies may arise from this development.

In the following, Section 2 reviews some proposals for the electrical model employed for the biological-electrode characterization, and the parameter related with. Section 3 summarizes the techniques developed for impedance measurement. Section 4 introduces the circuit issues related with system portability, lab-on-chip approaches. Section 5 depicts some application using bioimpedance measurements. Finally, Section 6 summarizes the work conclusions.

\section{Bio-electrode Electrical Model}

Bio-electrodes employed to signal management on impedance acquisition modify the voltage and current involved in test, due to their electrical response according with their electrical model. These models can change depending on the kind of sensor: resistive or capacitive, and load involved in the application: saline solution, medium, cells, tissues, etc. under test. So, one of the first steps to solve in BI LoC design is to define the electrical electrode model for each application

The impedance of electrodes in ionic liquids has been described quite extensively [50-53]. When a solid (including metals, semiconductors, and insulators) is immersed in an ionic solution, ions in the solution can react with the electrode and the solid ions from the electrode can enter the solution, leading to complex reactions at the interface. An electrified interface or double layer develops at the interface of the two phases. Eventually, electrochemical equilibrium is established at the interface: the current flowing into the electrode is equal but opposite in sign to that flowing out from the electrode. The net result is the development of a charge distribution at the interface with an associated electric potential distribution. The Helmholtz-Gouy-Chapman-Stern model is the commonly accepted model for describing the charge distribution at the electrode interface [54]. The negatively charged electrode attracts hydrated ions with positive charges to the surface but repels negatively charged ions away from the surface, yielding the profiles of cation and anion concentration $\mathrm{C}+$ and $\mathrm{C}-$, respectively. The water dipoles are also reoriented under the electric fields. Some ionic species 
that are not obstructed by their primary hydration sheath, such as some anions, can make their way to and come into the electrode. The charge distribution extends to the bulk solution thanks to thermal motion, forming an ion cloud-like diffusion layer and a charge spatial distribution. The profile of the diffuse zone depends on the Debye length, which in turn depends on the fluid constant, the temperature, the ion charge number and the ion concentration of the bulk solution [26].

When a small enough sinusoidal current is applied to the electrode at equilibrium, the electrode potential will be modulated by a sinusoidal overpotential [55]. In the range of linear behavior, the phasor ratio of the output overpotential to the input current defines the AC polarization impedance. During the small current perturbation, a charge transfer due to chemical reactions and mass diffusion occurs at the electrode surface. The rate-determining step will dictate the electrode polarization impedance.

In the following paragraph, we will first discuss an equivalent circuit representing all the phenomena occurring at the electrode-solution interface and, then, we will explain each component in the circuit. The electrified interface can be considered as the series connection of two parallel-plate capacitors with the thicknesses of a compact layer and a diffuse layer respectively, with a water dielectric. This is the electrode-solution interfacial capacitance of the electrified double layer. Apart from the double layer capacitance, $\mathrm{C}_{\mathrm{I}}$, the electrode-solution interface has faradic impedance representing a barrier to current flow between the electrode and the solution, including the finite rate of mass transport and electron transfer at the electrode surface. These phenomena are modeled in the equivalent circuit in Fig. 2, in which the faradic impedance is in parallel with the double layer capacitance. The current flowing through the electrified interface will encounter a resistance $R_{c t}$ caused by the electron transfer at the electrode surface and Warburg impedance $Z_{W}$ due to limited mass diffusion from the electrode surface to the solution. As a result, in the equivalent, the electron transfer resistance $R_{c t}$ is in series with the mass diffusion limited impedance $Z_{\mathrm{W}}$. As the current spreads to the bulk solution, the electrode has a solution conductivity, determined series resistance, represented as spreading resistance $R_{S}$ in the equivalent circuit.

The circuit in Fig. 2 describes, in a general sense, most of electrode performances. In some applications, there are only capacitive path signal between the electrode-sample, so $\mathrm{R}_{\mathrm{ct}}$ resistance can be rejected. At some others, the model must be matched to the biological sampled being measure, as for example, measuring impedance of tissues [23], cell cultures [29], or cell density in suspension 3D [27], due to particular relationship observed between the electrodes and the bio-samples. In the electrode biosample modelling field, alternative setups and methodologies for LoC system are currently being explored [3].

The derivation of exact and confident models is an open task today, in order to increase the degree of knowledge of involved processes from measurement performed [25], and many works look forward to obtain cell signatures as a way to detection (cancer, toxicology responses, stem cells, liver-blood, myocardial cells). Furthermore, in Ref. [27] it is proposed the use of electrical model for cell suspension (3D) assays instead of the model employed by ECIS based on cell attachment (2D) to substrate, as a way to better imitate at micro labs the real performances of assays in tissues and organs. Several modelling techniques are being used for single cells and monolayers electrical models synthesis. In Ref. [30], a

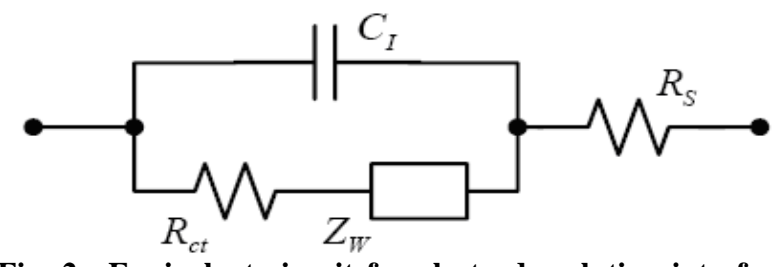

Fig. 2 Equivalent circuit for electrode solution interface. $\mathrm{C}_{\mathrm{I}}$ is the double layer capacitance; the Faradic impedance includes $Z_{X}$, the Warburg impedance and $R_{c t}$, the charge-transfer resistance. $R_{S}$ is the spreading resistance. 
set of three parameters were proposed: $R_{b}$, barrier resistance between monolayer cells, $h$, gap distance from cell to attached substrate, and $r$, proposed cell radius, as a way to solve the electromagnetic field equations at the cell-to-electrode interface, by fitting these parameters to measurements. Another approach employs the finite element simulations, as COMSOL $[28,56]$ as a way to reproduce the electric conditions at the sensing structure for solving the electromagnetic equations. The knowledge of these models makes possible the measure interpretation in terms of cell size, number, tissue impedance and other properties, enabling their incorporation to computer aided design tools for predictions, circuit design help, and reconstruction data $[57,58]$.

\section{Bio-impedance Measurement Techniques}

Commonly, these techniques require excitation and processing circuits. Excitation is usually done with AC sources: current or voltages, with the objective of measure the response of $Z_{x}$. To define the technique to be applied, it must be evaluated first the electrode setup, its electrical performance and influence on the circuits specifications, and second, the algorithm to extract magnitude and phase (or Real and Imaginary components).

In Ref. [48], processing steps are based on coherent demodulation principle, while synchronous sampling can be also employed [49], leading to good results. In both references, processing circuits must be synchronized with input signals, as a requirement for the technique to work, obtaining the best noise performance when proper filter functions (HP and LP) are incorporated. Block diagrams for it is illustrated in Fig. 3. The main drawback for in Ref. [48] is that the separated channels for in-phase and quadrature components must be matched to avoid large phase errors. Synchronous sampling proposed in Ref. [49] avoids two channels and demodulation, by selecting accurate sampling times, and adding a high pass filter in the signal path to prevent low-frequency noise and sampler interferences. These measurement principles work as feed-forward systems: the signal generated on $Z_{x}$ is amplified and then processed. Also, in Refs. [29, $40]$ it is proposed a simple solution based on a resistance to recover the in-phase reference signal to extract the full impedance components of $Z_{x}$. Another realization employs the computational power resources of a microprocessor to minimize the analogue part of the signal processing circuits, promoting the Amplitude-to-Time conversion procedure [59], taking advantage from the high frequency ( $300 \mathrm{MHz}$ ) clock existing in the $\mu \mathrm{P}$.

Among the feedback sensing techniques (Fig. 3), it can be found the closed loop [60] and the OB (Oscillation Based) approach [29]. These have the advantage of controlling the voltage applied to electrodes for linear biasing, and the possibility of perform the measurement thanks to the feedback loop control signal. In the $\mathrm{OB}$ are eliminated also the external AC excitation source, requiring only a DC power supply.

Two- and four-electrode represent the most commonly set-ups used to acquire BI signals. In two-wire approach [61], signal path incorporates the electrode response, while in four-wires this influence is cancelled or minimized. In three electrodes realizations, it is incorporated a $\mathrm{RE}$ (Reference Electrode), also with the CE (Counting Electrode) for measure and the WE (Working Electrode) for excitation. To summarize the techniques reviewed in this section, Table 3 is presented. The main techniques existing on the literatures are analysed below.

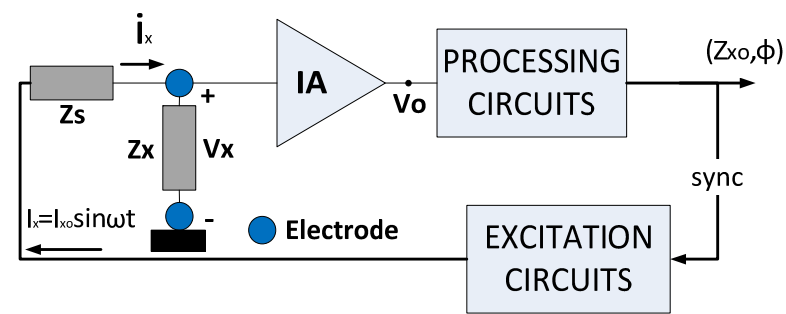

Fig. 3 General block diagram for the measurement of $Z_{x}$ impedance with a feedback configuration. 
Table 3 Bioimpedance measurement techniques.

\begin{tabular}{llll}
\hline BI Technique & Type & Sync. & AC Source \\
\hline Coherent demodulation & Feedforward & $\mathrm{X}$ & $\mathrm{X}$ \\
Synchronous sampling & Feedforward & $\mathrm{X}$ & $\mathrm{X}$ \\
Closed loop & Feedback & & $\mathrm{X}$ \\
OB (Oscillation Based) & Feedback & & \\
\hline
\end{tabular}

\section{System Portability Issues}

The reduction of cost, size, and weight; the increasing of the test performance; and the system wearability, are properties being demanded by the new biomedical generation systems. These systems are developed from recent biomedical advances and aims at increasing the life quality and healthcare systems: The LoC devices. These systems [3, 46] try to optimize classical assays and biomedical techniques, incorporating as main property the portability. Final application must be driven by the biologist or physician, answering the questions of why, how and what is a LoC implemented for. Point-of-Care, implanted, real time monitoring and supervision, drug delivery, lab assays, and so should be supervised by an expert, to define the main requirements. Actuation, sensing and electronics are the three main functional parts of LoC, which must follow several guidelines:

To preserve the bio-sample environment. Electrical signals values and ranges should be first (V/I) limited by living bodies, organs and cells. Electrical signal must preserve the sample quality, life and normal operation. This is not only a result from LoC develop process, but also, the bio assays, as for example, the 3D cell suspension assays, which seems to be a more realistic representation of the living cell environment in bodies, organs and tissues [27].

Low size/weight is mandatory for portability. This is currently being achieved with several micro-technologies (mechanical, optical, fluidic, and electronic, etc.) [3].

LP (Low-Power) is a compulsory requirement for wearable applications: electronics circuits must be designed to optimize power consumption. Pacemakers are examples of the first implanted apparatus in the body with a large history and evolution developed so far. Together, a power management unit should be incorporated. Low-Voltage specification can be relevant, but usually is considered in second place after low power consumption.

Remote sensing and real time monitoring of process and biomarkers can be enabled with wireless or RF communication units. Microelectronics technology makes this option possible, enabling the reduction of the power consumption of the wireless system. In addition, the correct selection of the communication protocols will impact over the power consumption of the LoC. Commercial solutions based on numerous protocols are available.

\section{Applications}

Many application fields are being inspired by the LoC development. Medical (diagnosis, therapeutic, clinical, research, drug development), Biology (cell-culture: grow, toxicology, cancer, stems cells; PCR, etc.) and Industry (food control, bacterial, agriculture), are some examples, and in many cases, there is not a real border between all of them. Bio-impedance measurement performed with LoC systems are being applied to many areas: biology assays including Cell-Culture are being performed in Ref. [27], where the 2D cell-culture test principle developed by ECIS [30] is generalized to 3D systems, through the spheroidal impedance concept. This responds to the hypothesis that living cells in bodies, tissues and organs are in this 3D environment, not attached to substrate as in classical cell cultures. The analysis is based on constant phase models proposed for the microelectrode-medium-cells in the microsystem developed, and is applied to stem cells 
differentiation and high throughput drug testing assays. Similar analysis is performed in Ref. [32] by proposing an electrical model of the cell cancer lines in suspension. Bioimpedance electrical parameters search for the microelectrodes-cells applied to HeLa cancer cell line is proposed in Ref. [31]. This work tries to find a "signature" of this cell lines based on the parameter associated to the electrode-cell model, by using a commercial impedance analyser. Cell cytotoxicity is studied in Ref. [62] with several electrode sizes, in order to define a breast cancer cell-signature respect to a proposed model. Assays for cell growth, attachment and spreading are reported using ECIS technique.

In classic medical environments, like diagnosis, therapeutic and clinics, it can be found several BI based approaches. A four-wire setup is employed in Ref. [42] for ECG and hydration studies in human body using BI, with capacitive electrodes through the textile material and clothes. The electronics platform proposed is reported. Myocardial ischemic reversibility is studied in Ref. [22], by applying two Iridium electrodes with low polarization effects. The objective is to define how infarcted myocardial cells can be recovered with success after an ischemia happens. Body Skin Bioimpedance for EIT application in neonatal function monitoring is the challenge in microsystem presented in Ref. [63]. A 1.5 $\mu \mathrm{m}^{2}$ full microelectronic system is developed, with synchronous demodulation and multi-frequency capability. CRT (Cardiac Resynchronization Therapy) requires accurate heart rate provided by real time monitoring to correctly program the rate of stimulation for pacemaker devices. A fully integrated system with LP consumption $(13 \mu \mathrm{A})$, synchronous demodulation and ECG and respiration rate acquisition is reported in Ref. [41]. Also, with the help of a defibrillator as instrument, respiratory rate can be obtained [44]. In Ref. [45] it is proposed the measurement of the PWV (Pulse Wave Velocity) of blood in the human body by studying the delay in the blood signal path from heart to wrist. For that, two sensors are required: one ECG at the chest, and other $\mathrm{BI}$ at the wrist for blood flow rate measurement. An active-electrode is being proposed for "in-situ" picking-up the EEG signals to improve the signal quality in EIT brain imaging applications. The in-situ signal acquisition allows the better rejection of noise signal and CMRR reduction effects from electrode and other element mismatching [43]. Models for cortex cranial tissue are being developed to establish the tissue reaction to post-implantation EEG sensors [23], and also for electrical stimulation devices focused on neural activity rehabilitation. Glucose concentration in blood [36], body hydration etc., together with some industrial application focused in food test, like bacterial detection using inter-digit sensor presented in Ref. [15] and other examples.

Between the non-specific application and those focused on computer aid applications, we can find the followings. A BI emulator is proposed in Ref. [57] with the aims of reproduce biological response in EIT experiments [39]. This represents an alternative to classical direct reconstruction problem or finite element approaches. The $\mathrm{P}$ capabilities are exploited in Ref. [59] to minimize the specific analogue and digital circuitry required and increases the SNR (signal-to-noise ratio) for BI measurements in EIT. It is implemented an amplitude-to-time converter by direct high frequency sampling based on microprocessor clock rate. Errors in coherent demodulation approach are analysed in Ref. [64], encouraging a mixed sinusoidal-squared trade-offs for excitation and demodulation processes that allows error correction and increases the BI measurement accuracy. Development of an electrical model for spinal cord stimulations is presented in Ref. [18], by extending the linear model of the electrode, to large voltage amplitudes in which the non-linear performance of transfer resistance $\left(\mathrm{R}_{\mathrm{CT}}\right)$ is dominant. A Liver tissue electrical-model proposal is described in Ref. [24] to study the blood-flow in contrast to laser 
Doppler flow sensors. This hypothesis correlates the haematocrit blood cells with the electrical model of the liver tissue.

Important mention is deserved by the so-called POC (Point-of-Care) technology. This technology would enable the health systems to achieve a higher level of quality in patient care services. Not to mention the importance of having a whole family of cheap and robust devices capable of diagnosing a great variety of conditions in the under-developed countries. The tendency described in this article towards the development of Laboratory-On-Chip enabled devices could possibly lead to the future technology in healthcare sector of PoC systems based on bioimpedance measurements. Furthermore, as we approach (and interact with) the biophysical scale at which most biochemical reactions occur, new methodologies may arise that may help to improve our understanding of the biochemistry and provide new technologies for better diagnose and therapy. All of this can be developed thanks to the microelectronic technology.

Finally, continuous monitoring of health variables in a non-invasive, cheap and effective way can lead to a deep transformation in the way healthcare services are applied. Possibility of having not only current value for patient health variables; blood biochemistry, temperature, blood cell count, heart rate, pressure, breath monitoring, but the historical data, is to be provided by using this technology and applying the novel concepts of "Wearable" technology and "The IoT (Internet of Things)" [11, 17].

\section{Conclusions}

The use of bioimpedance parameter as biomarker, for characterization of many medical and biological processes, has been reviewed considering various reference points. From electrode point of view, the knowledge of the electrode performance below electrical excitation is mandatory for the success of designed circuits and data decoding. Each measurement situation corresponds to a kind of electrode and responds to characteristic impedance depending from sample being tested. Bio-electrode electrical model knowledge and its limits must be defined for each particular case. Measurement techniques based on electronic circuit implementation must consider specifications imposed by electrodes.

The recommended choice is the OB (Oscillation Based) approach, which lacks requisite for synchronization between excitation-response signals and empowers the system with higher control on the excitation signal. Circuit design should consider also the low voltage level of many signals involved (SNR) and channel mismatch (CMRR for amplifiers). System portability is today a challenge for many biomedical applications. Limitation of signal ranges, reduced weight and size, low power consumption and wireless communication should be rules for driving the LoC system and its corresponding circuit design. Many of the current implementations are not really portable; trying to identify and correlate the bioimpedance measurements with biomedical processes, with the electronic not yet being incorporated to the biomedical lab.

\section{Acknowledgment}

This work was supported in part by the Spanish founded Project: TEC 2013-46242-C3-1-P: Integrated Microsystem for Cell Culture Assays, co-finance with FEDER.

\section{References}

[1] Harrison, D. J., Fluri, K., Seiler, K., Fan, Z., Effenhauser, C. S., and Manz, A. 1993. "Micromachining a Miniaturized Capillary Electrophoresis-Based Chemical Analysis System on a Chip." Science 261 (5123): 895-7.

[2] Oosterbroek, R., and Berg, A. 2003. Lab-on-a-Chip. Miniaturised Systems for (Bio) Chemical Analysis and Synthesis. Elsevier B.V.

[3] Ghallab, Y. H., and Ismail, Y. 2014. "CMOS Based Lab-on-a-Chip: Applications, Challenges and Future Trends" IEEE Circuits Syst. Mag. 14 (2): 27-47.

[4] Romani, A., Manaresi, N., Marzocchi, L., Medoro, G., 
Leonardi, A., Altomare, L., Tartagni, M., and Guerrieri, R. 2004. "Capacitive Sensor Array for Localization of Bioparticles in CMOS Lab-on-a-chip." 2004 IEEE International Solid-State Circuits Conference (IEEE Cat. No.04CH37519), 4-11.

[5] Medoro, G., Manaresi, N., Leonardi, A., Altomare, L., Tartagni, M., and Guerrieri, R. 2003. "A Lab-on-a-chip for Cell Detection and Manipulation." IEEE Sens. J. 3 (3): 317-25.

[6] Manaresi, N., Romani, A., Medoro, G., Altomare, L., Leonardi, A., Tartagni, M., and Guerrieri, R. 2003. "A CMOS Chip for Individual Cell Manipulation and Detection.” 2003 IEEE International Solid-State Circuits Conference, Digest of Technical Papers ISSCC., Vol. 1, 192-487.

[7] Yúfera, A., Gallego, E., and Molina, J. 2011. "ImagCell: A Computer Tool for Cell Culture Image Processing Applications in Bioimpedance Measurements." Advances in Experimental Medicine and Biology 696: 733-40.

[8] Piacentini, N., Demarchi, D., Civera, P., and Knaflitz, M. 2008. "MEMS-Based Blood Cell Counting System." Electronics, Circuits and Systems, 2008. ICECS 2008. 15th IEEE International Conference, 198-201.

[9] Majer, L., Stopjakov, V., and Vavrinsk, E. 2009. "Wireless Measurement System for Non-Invasive Biomedical Monitoring of Psycho-Physiological Processes." J. Electr. Eng. 60 (2): 57-68.

[10] Nelson, N., Sander, D., Dandin, M., Prakash, S. B., Sarje, A., and Abshire, P. 2009. "Handheld Fluorometers for Lab-on-a-chip Applications." IEEE Trans. Biomed. Circuits Syst. 3 (2): 97-107.

[11] Holzinger, A. 2014. Biomedical Informatics: Discovering Knowledge in Big Data. Cham: Springer International Publishing.

[12] Satake D., Ebi, H., Oku, N., Matsuda, K., Takao H., et al. 2002. "A Sensor for Blood Cell Counter Using MEMS Technology." Sensors Actuators, B Chem. 83 (1-3): 77-81.

[13] Yúfera, A., Rueda, A., Muñoz, J. M., Doldán, R., Leger, G., and Rodríguez-Villegas, E. O. 2005. "A Tissue Impedance Measurement Chip for Myocardial Ischemia Detection." IEEE Trans. Circuits Syst. I Regul. Pap. 52 (12): 2620-8.

[14] Yufera, A., and Rueda, A. 2009. "A CMOS Bio-Impedance Measurement System." Advances in Experimental Medicine and Biology 696: 252-7.

[15] Radke, S. M., and Alocilja, E. C. 2004. "Design and Fabrication of a Microimpedance Biosensor for Bacterial Detection." IEEE Sens. J. 4 (4): 434-40.

[16] Gensler, H., Sheybani, R., Li, P. Y., Lo, R., Zhu, S.,
Yong, K. T., Roy, I., et al. 2010. "Implantable MEMS Drug Delivery Device for Cancer Radiation Reduction." Proceedings of the IEEE International Conference on Micro Electro Mechanical Systems (MEMS), 23-6.

[17] Chung, W. Y., Bhardwaj, S., Purwar, A., Lee, D. S., and Myllylae, R. 2007. "A Fusion Health Monitoring Using ECG and Accelerometer Sensors for Elderly Persons At Home." Annual International Conference of the IEEE Engineering in Medicine and Biology Proceedings, 3818-21.

[18] Scott, J., and Single, P. 2013. "Compact Nonlinear Model of an Implantable Electrode Array for Spinal Cord Stimulation (SCS)." IEEE Trans. Biomed. Circuits Syst. 8 (3): 382-90.

[19] Lajunen, T., Viitala, L., Kontturi, L. S., Laaksonen, T., Liang, H., Vuorimaa-Laukkanen, E., Viitala, T., Le Guèvel, X., Yliperttula, M., Murtomäki, L., and Urtti, A. 2015. "Light Induced Cytosolic Drug Delivery from Liposomes with Gold Nanoparticles." J. Control. Release 203: 85-98.

[20] Yao, H., Shum, A. J., Cowan, M., Lähdesmäki, I., and Parviz, B. A. 2011. "A Contact Lens with Embedded Sensor for Monitoring Tear Glucose Level." Biosens. Bioelectron. 26 (7): 3290-6.

[21] Schwan, H. P. 1994. "Electrical Propertis of Tissues and Cell Suspensions: Mechanisms and Models." Engineering in Medicine and Biology Society: 70-1.

[22] Mellert, F., Winkler, K., Schneider, C., Dudykevych, T., et al. 2011. "Detection of (Reversible) Myocardial Ischemic Injury by Means of Electrical Bioimpedance." IEEE Trans. Biomed. Eng. 58 (6): 1511-8.

[23] Mercanzini, A., Colin, P., Bensadoun, J.-C., Bertsch, A., and Renaud, P. 2009. "In Vivo Electrical Impedance Spectroscopy of Tissue Reaction to Microelectrode Arrays." IEEE Trans. Biomed. Eng. 56 (7): 1909-18.

[24] Huang, W. H., Chui, C. K., Teoh, S. H., and Chang, S. K. Y. 2012. "A Multiscale Model for Bioimpedance Dispersion of Liver Tissue.” IEEE Trans. Biomed. Eng. 59 (6): 1593-7.

[25] Daza, P., Olmo, A., Cañete, D., and Yúfera, A. 2013. "Monitoring Living Cell Assays with Bio-impedance Sensors." Sensors Actuators B Chem. 176: 605-10.

[26] Borkholder, D. 1998. "Cell Based Biosensors Using Microelectrodes.” Stanford University.

[27] Alexander, F. A., Price, D. T., and Bhansali, S. 2013. "From Cellular Cultures to Cellular Spheroids: Is Impedance Spectroscopy a Viable Tool for Monitoring Multicellular Spheroid (MCS) Drug Models?" IEEE Rev. Biomed. Eng. 6: 63-76.

[28] Huang, X., Nguyen, D., Greve, D. W., and Domach, M. M. 2004. "Simulation of Microelectrode Impedance 
Changes Due to Cell Growth." IEEE Sens. J. 4 (5): 576-83.

[29] Huertas, G., Maldonado, A., Yufera, A., Rueda, A., and Huertas, J. L. 2015. "The Bio-oscillator: A Circuit for Cell-Culture Assays." IEEE Trans. Circuits Syst. II Express Briefs 62 (2): 164-8.

[30] Giaever, I., and Keese, C. R. 1986. "Use of Electric Fields to Monitor the Dynamical Aspect of Cell Behavior in Tissue Culture." IEEE Trans. Biomed. Eng. 33 (2): 242-7.

[31] Das, D., Kamil, F. A., Agrawal, S., Biswas, K., and Das, S. 2014. "Fragmental Frequency Analysis Method to Estimate Electrical Cell Parameters From Bioimpedance Study.” IEEE Trans. Instrum. Meas. 63 (8): 1991-2000.

[32] Guo, F. Q., Wei, W., Wei, D., Fan, Zh., Sinclair, A. J., and Chatwin, C. R. 2012. "Bioimpedance Analysis for the Characterization of Breast Cancer Cells in Suspension." IEEE Trans. Biomed. Eng. 59 (8): 2321-9.

[33] Aberg, P., Nicander, I., Hansson, J., Geladi, P., Holmgren, U., and Ollmar, S. 2004. "Skin Cancer Identification Using Multifrequency Electrical Impedance-A Potential Screening Tool." IEEE Trans. Biomed. Eng. 51 (12): 2097-102.

[34] Giaever, I., and Keese, C. R. 1991. "Micromotion of Mammalian Cells Measured Electrically." Proc. Natl. Acad. Sci. 88 (17): 7896-900.

[35] Linderholm, P., Braschler, T., Vannod, J., Barrandon, Y., Brouard, M., and Renaud, P. 2006. "Two-dimensional Impedance Imaging of cell Migration and Epithelial Stratification." Lab Chip 6 (9): 1155-62.

[36] Beach, R. D., Conlan, R. W., Godwin, M. C., and Moussy, F. 2005. "Towards a Miniature Implantable In Vivo Telemetry Monitoring System Dynamically Configurable as a Potentiostat or Galvanostat for Twoand Three-Electrode Biosensors." IEEE Trans. Instrum. Meas. 54 (1): 61-72.

[37] Manickam, A., Chevalier, A., McDermott, M., Ellington, A. D., and Hassibi, A. 2010. "A CMOS Electrochemical Impedance Spectroscopy (EIS) Biosensor Array." IEEE Trans. Biomed. Circuits Syst. 4 (6): 379-90.

[38] Hassibi, A., and Lee, T. H. 2006. "A Programmable 0.18- $\mu \mathrm{m}$ CMOS Electrochemical Sensor Microarray for Biomolecular Detection.” IEEE Sens. J. 6 (6): 1380-8.

[39] Holder, D. 2005. Electrical Impedance Tomography: Methods, History and Applications. IOP.

[40] Ausin, J. L., Ramos, J., Torelli, G., and Duque-Carillo, J. F. 2014. "Live Demonstration: A Wireless Multichannel Bioimpedance Spectrometer for Patient Monitoring." 2014 IEEE Biomedical Circuits and
Systems Conference (BioCAS) Proceedings, 176.

[41] Yan, L., Pettine, J., Mitra, S., Kim, S., Jee, D.-W., Kim, H., Osawa, M., Harada, Y., Tamiya, K., Van Hoof, C., and Yazicioglu, R. F. 2013. "A $13 \mu$ A Analog Signal Processing IC for Accurate Recognition of Multiple Intra-cardiac Signals." IEEE Trans. Biomed. Circuits Syst. 7 (6): 785-95.

[42] Taji, B., Shirmohammadi, S., Groza, V., and Batkin, I. 2014. "Impact of Skin-Electrode Interface on Electrocardiogram Measurements Using Conductive Textile Electrodes.” IEEE Trans. Instrum. Meas. 63 (6): 1412-22.

[43] Guermandi, M., Cardu, R., Franchi Scarselli, E., and Guerrieri, R. 2015. "Active Electrode IC for EEG and Electrical Impedance Tomography with Continuous Monitoring of Contact Impedance." IEEE Trans. Biomed. Circuits Syst. 9 (1):21-33.

[44] Martinsen, Ø. G., Nordbotten, B., Grimnes, S., Fossan, H., and Eilevstjønn, J. 2014. "Bioimpedance-Based Respiration Monitoring with a Defibrillator." IEEE Trans. Biomed. Eng. 61 (6): 1858-62.

[45] Lee, W., and Cho, S. 2015. "Integrated All Electrical Pulse Wave Velocity and Respiration Sensors Using Bio-Impedance." IEEE J. Solid-State Circuits 50 (3): 776-85.

[46] Ha, S., Kim, C., Chi, Y. M., Akinin, A., Maier, C., Ueno, A., and Cauwenberghs, G. 2014. "Integrated Circuits and Electrode Interfaces for Noninvasive Physiological Monitoring." IEEE Trans. Biomed. Eng. 61 (5): 1522-37.

[47] Grimnes, S., and Martinsen, O. 2008. Bioimpedance and Bioelectricity Basics. Academic Press.

[48] Ackmann, J. J. 1993. "Complex Bioelectric Impedance Measurement System for the Frequency Range From 5 Hz to 1 MHz." Ann. Biomed. Eng. 21 (2): 135-46.

[49] Pallás-Areny, R., and Webster, J. G. 1993. "Bioelectric Impedance Measurements Using Synchronous Sampling.” IEEE Trans. Biomed. Eng. 40 (8): 824-9.

[50] Robinson, D. A. 1968. "The Electrical Properties of Metal Microelectrodes." Proc. IEEE 56 (6): 1065-71.

[51] Schwan, H. P. 1963. Electrophysiological Methods. Elsevier.

[52] Simpson, R. W., Berberian, J. G., and Schwan, H. P. 1980. "Nonlinear AC and DC Polarization of Platinum Electrodes.” IEEE Trans. Biomed. Eng. 27 (3): 166-71.

[53] Schwan, H. P. 1992. "Linear and Nonlinear Electrode Polarization and Biological Materials." Ann. Biomed. Eng. 20 (3): 269-88.

[54] Bockris, J. O., and Reddy, A. K. N. 1970. Modern Electrochemistry. Plenum Press.

[55] Schwan, H. P. 1968. "Electrode Polarization Impedance and Measurements in Biological Materials." Ann. N. Y. 
Acad. Sci. 148 (1): 191-209.

[56] COMSOL. "The Use of the Conductive Media DC Application Mode for Time-Harmonic Problems, Solution."

[57] Li, N., Xu, H., Zhou, Z., Xin, J., Sun, Z., and Xu, X. 2013. "Reconfigurable Bioimpedance Emulation System for Electrical Impedance Tomography System Validation.” IEEE Trans. Biomed. Circuits Syst. 7 (4): 460-8.

[58] Ramos, J., Ausin, J. L., Torelli, G., and uque-Carrillo, J. F. 2009. "A Wireless Sensor Network for Fat and Hydration Monitoring by Bioimpedance Analysis." In Proceedings of the 6th International Workshop on Wearable, Micro, and Nano Technologies for Personalized Health, 49-52.

[59] Baeg, J. C., Wi, H., Oh, T. I., McEwan, A. L., and Woo, E. J. 2013. "An Amplitude-to-time Conversion Technique Suitable for Multichannel Data Acquisition and Bioimpedance Imaging." IEEE Trans. Biomed. Circuits Syst. 7 (3): 349-54.

[60] Yúfera, A., and Rueda, A. 2010. "Design of a CMOS Closed-loop System with Applications to
Bio-impedance measurements." Microelectronics J. 41 (4): 231-9.

[61] Bragós, R., Sarro, E., Fontova, A., Soley, A., Cairó, J., Bayés-Genís, A., and Rosell, J. 2006. "Four Versus Two-electrode Measurement Strategies for Cell Growing and Differentiation Monitoring Using Electrical Impedance Spectroscopy." Conf. Proc. Annu. Int. Conf. IEEE Eng. Med. Biol. Soc. IEEE Eng. Med. Biol. Soc. Annu. Conf. 1: 2106-9.

[62] Pradhan, R., Mandal, M., Mitra, A., and Das, S. 2014. "Assessing Cytotoxic Effect of ZD6474 on MDA-MB-468 Cells Using Cell-Based Sensor.” IEEE Sens. J. 14 (5): 1476-81.

[63] Kassanos, P., Constantinou, L., Triantis, I. F., and Demosthenous, A. 2014. "An Integrated Analog Readout for Multi-Frequency Bioimpedance Measurements.” IEEE Sens. J. 14 (8): 2792-800.

[64] Kubendran, R., Lee, S., Mitra, S., and Yazicioglu, R. F. 2014. "Error Correction Algorithm for High Accuracy Bio-impedance Measurement in Wearable Healthcare Applications.” IEEE Trans. Biomed. Circuits Syst. 8 (2): 196-205. 